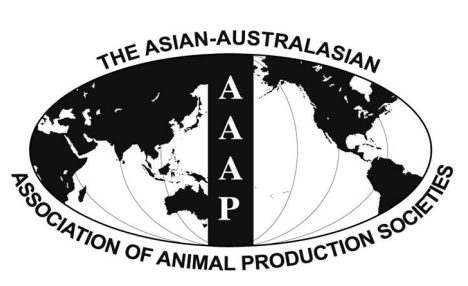

Open Access

Asian Australas. J. Anim. Sci.

Vol. 28, No. 9 : 1345-1353 September 2015

http://dx.doi.org/10.5713/ajas.14.0802

www.ajas.info

pISSN 1011-2367 elSSN 1976-5517

\title{
Influence of Rapeseed Meal on Growth Performance, Blood Profiles, Nutrient Digestibility and Economic Benefit of Growing-finishing Pigs
}

\author{
H. B. Choi, J. H. Jeong, D. H. Kim, Y. Lee ${ }^{1}$, H. Kwon ${ }^{1}$, and Y. Y. Kim* \\ Department of Agricultural Biotechnology, College of Animal Life Sciences, \\ Seoul National University, Seoul 151-742, Korea
}

\begin{abstract}
This study was conducted to investigate the influence of dietary rapeseed meal (RSM) on growth performance, blood profiles, nutrient digestibility and economic benefit of growing-finishing pigs. A total of 120 growing pigs ([Yorkshire $\times$ Landrace] $\times$ Duroc) with an initial body weight (BW) $29.94 \pm 0.06 \mathrm{~kg}$ were used in this experiment. Pigs were randomly allotted into 1 of 5 treatments in a randomized complete block design and 6 replicates with 4 pigs per pen. Treatments were divided by dietary RSM supplementation levels $(0 \%, 3 \%, 6 \%, 9 \%$, or $12 \%)$ in growing-finishing diets. A linear decrease $(\mathrm{p}<0.05)$ of BW and average daily gain (ADG) were observed at 13th wk of finishing and overall periods of pigs. Additionally, gain-to-feed ratio (G/F) tended to decrease by dietary RSM supplementation in growing-finishing diets (linear, $p=0.07$ and quadratic, $p=0.08$ ). Concentrations of serum triiodothyronine and thyroxine were not influenced by dietary RSM treatments whereas thyroid gland and liver weight were increased at 13th wk of finishing period (linear, $\mathrm{p}<0.05$; $\mathrm{p}<0.01$ ) by increasing dietary RSM supplementation level. In blood profiles, serum total cholesterol and low density lipoprotein cholesterol concentrations were not differed by dietary treatments at 13th wk of finishing period whereas concentration of serum high density lipoprotein cholesterol was affected by the supplementation level of RSM, resulting in a linear RSM level responses $(p<0.05)$. Serum blood urea nitrogen concentration tended to decrease (linear, $p=0.07 ; p=0.08$ ) at 6 th wk of growing and 13th wk of finishing periods and digestibility of dry matter tended to decrease by dietary RSM (linear, $p=0.09$ ). Crude protein, crude fat and nitrogen retention, whereas, were not affected by dietary RSM supplementation level. In the economic analysis, feed cost per weight gain was numerically decreased when RSM was provided up to $9 \%$. Consequently, RSM could be supplemented to growing-finishing diets up to $9 \%(3.07 \mu \mathrm{mol} / \mathrm{g}$ Gls $)$ without detrimental effects on growth performance of growing-finishing pigs. (Key Words: Rapeseed Meal, Growth Performance, Blood Profiles, Nutrient Digestibility, Growing-finishing Pigs)
\end{abstract}

\section{INTRODUCTION}

The cost of pig production is mainly affected by feed cost which impacts pork producers' profitability. Soybean meal (SBM) is the most widely used protein source throughout the world whereas increasing dietary supplementation level of SBM caused an increase feed cost in pig diets (Patrick et al., 2010; Smit et al., 2014). Rapeseed meal (RSM) is a by-product of rapeseed after removal of oil and is cost effective protein source in pig

\footnotetext{
* Corresponding Author: Y. Y. Kim. Tel: +82-2-880-4801, Fax: +82-2-878-5839, E-mail: yooykim@snu.ac.kr

${ }^{1}$ Department of Food and Nutrition, Seoul National University, Seoul 151-742, Korea.

Submitted Oct. 15, 2014; Revised Dec. 13, 2014; Accepted Jun. 2, 2015
}

diets and also alternative ingredient to SBM (Quiniou et al., 2012).

Rapeseed meal is generally contained $33 \%$ to $40 \%$ protein. The RSM has lower lysine $(5.5 \%$ vs $6.2 \%$ of protein) but higher methionine ( $2.1 \%$ vs $1.4 \%$ of protein) and cystine ( $1.2 \%$ vs $0.6 \%$ of protein) compares with SBM (Bell, 1984). However, RSM has higher crude fiber (14.1\% vs $3.9 \%$ ) than that of SBM which means RSM contains higher neutral detergent fiber (NDF). High NDF in RSM reduces apparently ileal digestibility of lysine $(74 \%$ vs $87 \%)$ and lowers protein digestibility ( $75 \%$ vs $90 \%)$ compared to SBM (Bell, 1984; Noblet and Goff, 2001) so nutrient digestibility may be attributed to elevated levels of fibers components (Patrick et al., 2010). Moreover, limited amount of RSM has been used in swine diets because of 
anti-nutritional factors such as glucosinolates (Gls) and erucic acid (Mawson et al., 1993a).

Hydrolysis products of Gls are toxic and may resulted in poor growth performance, enlarged thyroid gland, and reducing of circulating thyroid hormones (Mawson et al., 1994; Schöne et al., 1997). In addition, voluntary feed intake could be affected by inclusion of RSM in the diets because of decreased feed consumption due to high content of erucic acid, bitter taste of sinapine, and astringent effect in the mouth by tannin (Mawson et al., 1993b; Food Standards Australia New Zealand, 2003). Tripathi and Mishra (2007) demonstrated poor feed intake and reduced growth performance of pigs were observed when pigs were fed 1.3 to $2.8 \mu \mathrm{mol} / \mathrm{g}$ Gls diets. Therefore, the present study was conducted to determine the influence of dietary RSM levels in swine diets on growth performance, blood profiles, nutrient digestibility and economic benefit of growingfinishing pigs.

\section{MATERIALS AND METHODS}

\section{Animal management, experimental diets and housing}

A total of 120 growing pigs ([Yorkshire $\times$ Landrace] $\times$ Duroc; $29.94 \pm 0.06 \mathrm{~kg}$ initial body weight [BW]) were used in a 13 wk growth trial, at a research farm located in Suwon, South Korea. The pigs were allotted into five treatment groups based on their initial BW and sex with 6 replicates according to randomized completely block design. Five experimental diets containing different levels of RSM $(0$, $3 \%, 6 \%, 9 \%$, or $12 \%$ ) were provided to pigs during growing ( 0 to 6 th wk) and finishing ( 7 th to 13 th wk) periods. Each diet in growing period contained 3,250 kcal of $\mathrm{ME} / \mathrm{kg}$, $14.0 \%$ of crude protein $(\mathrm{CP})$ and $0.86 \%$ of total lysine and the assigned levels of RSM, respectively. The finishing period diet contained 3,261 kcal of $\mathrm{ME} / \mathrm{kg}, 11.49 \%$ of $\mathrm{CP}$ and $0.68 \%$ of total lysine. The RSM, used in current experiment, was imported from India. Solvent extract of the RSM (Table 1) was supplemented to the diets with replacement of corn, SBM, and soy oil. This RSM was solvent extracted by hexane to remove the remaining oil and then desolventing toasted at $90^{\circ} \mathrm{C}$ to $100^{\circ} \mathrm{C}$. All nutrients were met or exceeded NRC (1998) nutrient requirement estimates. The formula and chemical composition of experimental diets are presented in Tables 2 and 3.

All pigs were housed in an environmentally controlled building with half-slotted concrete floors in growing period $\left(1.26 \times 2.55 \mathrm{~m}^{2}\right)$ and finishing period $\left(1.60 \times 3.00 \mathrm{~m}^{2}\right)$. Each pen equipped with a feeder and a nipple drinker to provide water and mash feed ad-libitum during growing-finishing periods Body weight and feed intake were recorded at 0,6 , and 13 wks to calculate the average daily gain (ADG), average daily feed intake (ADFI) and gain-to-feed ratio
Table 1. Anti-nutritional factors contents and chemical composition of rapeseed meal (as dry matter basis)

\begin{tabular}{lc}
\hline Item & Rapeseed meal \\
\hline Analyzed chemical composition & \\
Crude protein (\%) & 35.99 \\
Essential amino acids (\% of protein) & \\
Arg & 8.64 \\
His & 3.30 \\
Ile & 4.28 \\
Leu & 5.23 \\
Lys & 5.51 \\
Met & 1.34 \\
Phe & 4.54 \\
Thr & 4.71 \\
Val & 4.65 \\
Non-essential amino acids (\% of protein) & \\
Ala & 5.22 \\
Asp & 8.37 \\
Cys & 0.40 \\
Glu & 20.61 \\
Gly & 6.54 \\
Pro & 8.61 \\
Ser & 5.16 \\
Tyr & 2.89 \\
Glucosinolates ( $\mu$ mol $/ g)$ & \\
Progoitrin & \\
Sinigrin & 0.24 \\
Gluconapin & 7.10 \\
Total glucosinolates & 26.81 \\
Erucic acid (mg/g) & 34.14 \\
\hline
\end{tabular}

(G/F ratio).

\section{Sample collections and analysis}

Blood samples were collected from 6 pigs from each treatment and were obtained via anterior vena cava at 6th wk of growing period and 13th wk of finishing period, respectively. Blood samples were centrifuged at 1,700 $\mathrm{g}$ at $4^{\circ} \mathrm{C}$ for 15 min (Eppendorf centrifuge 5810R, Hamburg, Germany) to separate serum. All samples of sera were stored at $-20^{\circ} \mathrm{C}$ until analysis.

Rapeseed meal, fecal and urinary samples were analyzed for dry matter (967.03; AOAC, 1990), CP (976.05; AOAC, 1990), ether extract (920.39; AOAC, 1990) and crude ash (942.05; AOAC, 1990).

Estimation of amino acid content in RSM was carried out according to Moore (1963) and AccQ-Tag regent kits were used (Waters, Milford, MA, USA). Performic acid was used in oxidizing amino acids and neutralized with sodium citrate dihydrate, and then hydrolyzed with $6 \mathrm{~N} \mathrm{HCl}$ for 24 $\mathrm{h}$ at $110^{\circ} \mathrm{C}$ to be liberated from the protein. Amino acids were analyzed on HPLC (Waters 486, USA). All separations were generated on NOVA-Pak $\mathrm{C}_{18}(4 \mu \mathrm{m})$ column (Waters, USA) with temperature controlled at $37^{\circ} \mathrm{C}$ and operated with flow rate of $1 \mathrm{~mL} / \mathrm{min}$. 
Table 2. The formula and chemical composition of experimental diets in growing period (as-fed basis)

\begin{tabular}{|c|c|c|c|c|c|}
\hline \multirow{2}{*}{ Item } & \multicolumn{5}{|c|}{ Rapeseed meal level in growing period (\%) ${ }^{1,2}$} \\
\hline & RSM 0 & RSM 3 & RSM 6 & RSM 9 & RSM 12 \\
\hline \multicolumn{6}{|l|}{ Ingredients $(\%)$} \\
\hline Corn & 79.82 & 78.90 & 77.92 & 77.01 & 76.03 \\
\hline Soybean meal, $46 \% \mathrm{CP}$ & 17.06 & 14.73 & 12.42 & 10.08 & 7.79 \\
\hline Rapeseed meal & 0.00 & 3.00 & 6.00 & 9.00 & 12.00 \\
\hline Soy oil & 0.16 & 0.54 & 0.95 & 1.32 & 1.72 \\
\hline Dicalcium phosphate & 1.38 & 1.25 & 1.17 & 1.05 & 0.95 \\
\hline Limestone & 0.62 & 0.63 & 0.63 & 0.64 & 0.64 \\
\hline L-lysine $\mathrm{HCl}$ & 0.33 & 0.33 & 0.33 & 0.33 & 0.32 \\
\hline DL-methionine & 0.08 & 0.06 & 0.03 & 0.02 & 0.00 \\
\hline Vitamin premix ${ }^{3}$ & 0.10 & 0.10 & 0.10 & 0.10 & 0.10 \\
\hline Mineral premix ${ }^{4}$ & 0.10 & 0.10 & 0.10 & 0.10 & 0.10 \\
\hline Salt & 0.30 & 0.30 & 0.30 & 0.30 & 0.30 \\
\hline Emulsifier & 0.05 & 0.05 & 0.05 & 0.05 & 0.05 \\
\hline Sum & 100.00 & 100.00 & 100.00 & 100.00 & 100.00 \\
\hline \multicolumn{6}{|l|}{ Chemical composition $(\%)^{5}$} \\
\hline ME (kcal/kg) & $3,250.10$ & $3,250.32$ & $3,250.40$ & $3,250.01$ & $3,250.27$ \\
\hline $\mathrm{CP}$ & 13.95 & 13.95 & 13.95 & 13.95 & 13.95 \\
\hline Lys & 0.86 & 0.86 & 0.86 & 0.86 & 0.86 \\
\hline Met & 0.26 & 0.26 & 0.26 & 0.26 & 0.26 \\
\hline $\mathrm{Ca}$ & 0.58 & 0.58 & 0.58 & 0.58 & 0.58 \\
\hline Total P & 0.51 & 0.51 & 0.51 & 0.51 & 0.51 \\
\hline
\end{tabular}

RSM, rapeseed mea; $\mathrm{CP}$, crude protein; ME, metabolizable energy.

${ }^{1}$ RSM 0, basal diet; RSM 3, basal diet $+3 \%$ RSM; RSM 6, basal diet+6\% RSM; RSM 9, basal diet $+9 \%$ RSM; RSM 12, basal diet $+12 \%$ RSM.

${ }^{2} \mathrm{Gls}$ content in the diets was equivalent to $0,1.02,2.04,3.07$, and $4.09 \mu \mathrm{mol} / \mathrm{for} 0 \%, 3 \%, 6 \%, 9 \%$, and $12 \%$ of RSM supplementation groups, respectively.

${ }^{3}$ Provided per kg of diet: vitamin A, 16,000 IU; vitamin $\mathrm{D}_{3}, 3,200 \mathrm{IU}$; vitamin E, $35 \mathrm{IU}$; vitamin $\mathrm{K}, 5 \mathrm{mg}$; rivofalvin, $6 \mathrm{mg}$; pantothenic acid, $16 \mathrm{mg}$; niacin, $32 \mathrm{mg}$; d-biotin, $128 \mu \mathrm{g}$; vitamin $\mathrm{B}_{12}, 20 \mu \mathrm{g}$.

${ }^{4}$ Provided per kg of diet: Fe, $281 \mathrm{mg}$; Cu, $288 \mathrm{mg}$; Mn, $49 \mathrm{mg}$; I, $0.3 \mathrm{mg}$; Se, $0.3 \mu \mathrm{g}$.

${ }^{5}$ Calculated values.

Glucosinolates were extracted from RSM with $2 \mathrm{~mL}$ of boiling methanol solution $(70 \% \mathrm{vol} / \mathrm{vol})$ and $200 \mu \mathrm{L}$ internal standard spike solution of glucotropaeolin (ChromaDex, Irvine, CA, USA) was added immediately (International Standards Organization, 1992) and extracted Gls were purified on aDEAE Sephadex A-25 anion exchange column (St. Louis, MO, USA). Three types of Gls in RSM were determined by using High Performance Liquid Chromatography (HPLC; Sunnyvale, CA, USA). Desulfo-glucosinolates were separated using a Synergi Fusion-RP 80A $(100 \times 3 \mathrm{~mm}, 4$ um, Phenomenex, CA, USA $)$ with a flow rate of $1 \mathrm{~mL} / \mathrm{min}$. Glucosinolates (progoitrin, sinigrin and gluconapin) were confirmed by a Finnigan LCQ Deca XP plus Ion Trap Mass Spectrometer system (Thermo Finnigan, CA, USA) which confirmed by LC-ESIMS in positive mode.

Erucic acid content in RSM was analyzed on a 7890 Agilent Gas Liquid Chromatograph (Agilent Technologies, Palo Alto, CA, USA) and equipped with flame ionization detector and the column was SP-2560 (i.d. $100 \mathrm{~m} \times 0.25 \mathrm{~mm}$ $\times 0.20 \mu \mathrm{m}$ film). Nitrogen was used as carrier gas, injector core temperature was $250^{\circ} \mathrm{C}$, detector temperature was $260^{\circ} \mathrm{C}$ and column temperature was programmed to begin at $170^{\circ} \mathrm{C}$ and then increase to $250^{\circ} \mathrm{C}$ then remained at $240^{\circ} \mathrm{C}$ for $40 \mathrm{~min}$. Chromatography was calibrated with a mixture of 37 different fatty acids (FAME 37; Supelco Inc., Bellefonte, PA, USA) and the standard contained fatty acids ranging from $\mathrm{C}_{4: 0}$ to $\mathrm{C}_{24: \ln 9}$ and samples were added $250 \mu \mathrm{l}$ of internal standard spike solution (Pentadecanoic acid; Sigma) by the method of AOAC (1990).

Serum triiodothyronine $\left(T_{3}\right)$ and thyroxine $\left(T_{4}\right)$ concentrations were measured by electrochemiluminescence immunoassay $\left(\mathrm{T}_{3}\right.$ and $\mathrm{T}_{4}$ Kits, Roche, Mannheim, Germany). Serum total cholesterol, low density lipoprotein (LDL) cholesterol and high density lipoprotein (HDL) cholesterol concentrations were analyzed using enzymatic colorimetric assay (Cholesterol Kit; LDL-C plus 2nd generation Kit; HDL-C plus 3nd generation Kit, Roche, Mannheim, Germany). Serum glucose and blood urea nitrogen (BUN) concentrations were analyzed using a kinetic UV assay (Glucose Hexokinase Kit; UREA/BUN Kit, Roche, Mannheim, Germany).

\section{Metabolic trial}

A total of 20 pigs $([$ Yorkshire $\times$ Landrace $] \times$ Duroc $)$ with an initial BW 31.14 \pm 0.36 were allotted to five treatments in 
Table 3. The formula and chemical composition of experimental diets in finishing period (as-fed basis)

\begin{tabular}{|c|c|c|c|c|c|}
\hline \multirow{2}{*}{ Item } & \multicolumn{5}{|c|}{ Rapeseed meal level in finishing period $(\%)^{1,2}$} \\
\hline & RSM 0 & RSM 3 & RSM 6 & RSM 9 & RSM 12 \\
\hline \multicolumn{6}{|l|}{ Ingredients $(\%)$} \\
\hline Corn & 86.83 & 85.87 & 84.94 & 83.95 & 82.99 \\
\hline Soybean meal, $46 \%$ CP & 10.53 & 8.20 & 5.87 & 3.57 & 1.25 \\
\hline Rapeseed meal & 0.00 & 3.00 & 6.00 & 9.00 & 12.00 \\
\hline Soy oil & 0.00 & 0.38 & 0.77 & 1.17 & 1.57 \\
\hline Dicalcium phosphate & 1.16 & 1.07 & 0.97 & 0.88 & 0.79 \\
\hline Limestone & 0.57 & 0.57 & 0.57 & 0.57 & 0.57 \\
\hline L-lysine $\mathrm{HCl}$ & 0.29 & 0.29 & 0.29 & 0.28 & 0.28 \\
\hline DL-methionine & 0.07 & 0.07 & 0.04 & 0.03 & 0.00 \\
\hline Vitamin premix ${ }^{3}$ & 0.10 & 0.10 & 0.10 & 0.10 & 0.10 \\
\hline Mineral premix ${ }^{4}$ & 0.10 & 0.10 & 0.10 & 0.10 & 0.10 \\
\hline Salt & 0.30 & 0.30 & 0.30 & 0.30 & 0.30 \\
\hline Emulsifier & 0.05 & 0.05 & 0.05 & 0.05 & 0.05 \\
\hline Sum & 100.00 & 100.00 & 100.00 & 100.00 & 100.00 \\
\hline \multicolumn{6}{|l|}{ Chemical composition $(\%)^{5}$} \\
\hline $\mathrm{ME}(\mathrm{kcal} / \mathrm{kg})$ & 3261.79 & 3261.36 & 3261.20 & 3261.33 & 3261.29 \\
\hline $\mathrm{CP}$ & 11.49 & 11.49 & 11.49 & 11.49 & 11.49 \\
\hline Lys & 0.68 & 0.68 & 0.68 & 0.68 & 0.68 \\
\hline Met & 0.24 & 0.24 & 0.24 & 0.24 & 0.24 \\
\hline $\mathrm{Ca}$ & 0.50 & 0.50 & 0.50 & 0.50 & 0.50 \\
\hline Total P & 0.46 & 0.46 & 0.46 & 0.46 & 0.46 \\
\hline
\end{tabular}

RSM, rapeseed mea; CP, crude protein; ME, metabolizable energy.

${ }^{1}$ RSM 0, basal diet; RSM 3, basal diet+3\% RSM; RSM 6, basal diet+6\% RSM; RSM 9, basal diet+9\% RSM; RSM 12, basal diet+12\% RSM.

${ }^{2} \mathrm{Gls}$ content in the diets was equivalent to $0,1.02,2.04,3.07$, and $4.09 \mu \mathrm{mol} /$ for $0 \%, 3 \%, 6 \%, 9 \%$, and $12 \%$ of RSM supplementation groups, respectively.

${ }^{3}$ Provided per kg of diet: vitamin A, 16,000 IU; vitamin $\mathrm{D}_{3}$, 3,200 IU; vitamin E, $35 \mathrm{IU}$; vitamin $\mathrm{K}, 5 \mathrm{mg}$; rivofalvin, $6 \mathrm{mg}$; pantothenic acid, $16 \mathrm{mg}$; niacin, $32 \mathrm{mg}$; d-biotin, $128 \mu \mathrm{g}$; vitamin $\mathrm{B}_{12}, 20 \mu \mathrm{g}$.

${ }^{4}$ Provided per kg of diet: Fe, $281 \mathrm{mg} ; \mathrm{Cu}, 288 \mathrm{mg}$; Mn, $49 \mathrm{mg}$; I, $0.3 \mathrm{mg} ; \mathrm{Se}, 0.3 \mu \mathrm{g}$.

${ }^{5}$ Calculated values.

a completely randomized design. Each pig was housed in an individual metabolic crate in a room of steady temperature $\left(27^{\circ} \mathrm{C}\right)$, controlled with a heating lamp. The experimental diets were supplied twice a day at 07:00 $\mathrm{h}$ and 19:00 $\mathrm{h}$ according to the rate of 2.0 times of the maintenance requirement for ME (NRC, 1998) based on initial BW of pigs. Water was provided ad libitum. After 6 days adaptation period, pigs were subjected to 5 days collection and $0.5 \mathrm{~g}$ of chromic oxide and $0.5 \mathrm{~g}$ of ferric oxide were used as initial and end marker, respectively. Collected excreta were stored at $-20^{\circ} \mathrm{C}$ during the collection period and dried $\left(65^{\circ} \mathrm{C}, 72 \mathrm{~h}\right)$ and ground $(2 \mathrm{~mm}$ screen, Wiley mill) for chemical analysis at the end of trial.

\section{Economic analysis}

Economic analysis was conducted to compare the feed cost for $1 \mathrm{~kg}$ weight gain. The feed cost per weight gain was calculated based on price of raw materials during the time of the experiment (May, 2013). In each phase, index was calculated from the relative ratio of the feed cost per weight gain to RSM 0 treatment.

$$
\begin{aligned}
& \text { Feed cost } / \text { weight gain }(\text { won } / \mathrm{kg}) \\
& =\frac{\text { Feed cost }(\text { won } / \mathrm{kg}) \times \text { Feed intake per head }(\mathrm{kg} / \mathrm{pig})}{\text { Weight gain per head }(\mathrm{kg} / \mathrm{pig})}
\end{aligned}
$$

\section{Statistical analysis}

Data were analyzed by analysis of variance for a completely randomized design using the general linear model procedure of SAS (SAS Institute, 2009). Least squares means were calculated for each independent variable. Orthogonal polynomial contrasts were used to determine linear and quadratic effects by increasing dietary RSM levels in gestation for all measurements of growing pigs. Individual animals were used as the experimental unit. Alpha level used for the determination of significance for all analysis was 0.05 and tendency for all analysis was $p>0.05$ and $p<0.10$.

\section{RESULTS}

Rapeseed meal used in this study contained progoitrin $(0.24 \mu \mathrm{mol} / \mathrm{g})$, sinigrin $(7.10 \mu \mathrm{mol} / \mathrm{g})$ and gluconapin $(26.81$ 
Table 4. Glucosinolates (Gls, $\mu$ mole/g) and erucic acid (mg/g) contents of eight different rapeseed meal (RSM) imported from India (dry matter basis)

\begin{tabular}{lccccc}
\hline \multirow{2}{*}{ Item } & \multicolumn{3}{c}{ Contents of glucosinolates $(\mu \mathrm{mole} / \mathrm{g})$} & \multirow{2}{*}{ Erucic acid $(\mathrm{mg} / \mathrm{g})$} \\
\cline { 2 - 4 } & Progoitrin & Sinigrin & Gluconapin & Total Gls & 3.50 \\
A & 0.45 & 14.49 & 54.73 & 69.67 & 5.09 \\
B & 0.33 & 10.13 & 38.15 & 48.60 & 5.75 \\
C & 0.38 & 11.67 & 41.76 & 53.81 & 4.86 \\
D & 0.31 & 10.13 & 35.36 & 45.79 & 4.62 \\
E & 0.31 & 9.58 & 33.86 & 43.75 & 2.84 \\
F & 0.38 & 10.33 & 37.94 & 48.65 & 4.35 \\
G & 0.40 & 11.66 & 41.88 & 53.94 & 5.23 \\
H & 0.38 & 10.28 & 36.64 & 47.30 & 4.53 \\
Average & 0.37 & 11.03 & 40.04 & 51.44 & \\
\hline
\end{tabular}

$\mu \mathrm{mol} / \mathrm{g}$ ), which resulted in $34.14 \mu \mathrm{mol} / \mathrm{g}$ of total Gls (Table 1). The erucic acid content in RSM was $6.29 \mathrm{mg} / \mathrm{g}$ of dry matter (DM) basis. Additionally, RSM, used in current experiment, contained $0.37 \mu \mathrm{mol} / \mathrm{g}$ of progoitrin, 11.03 $\mu \mathrm{mol} / \mathrm{g}$ of sinigrin and $40.04 \mu \mathrm{mol} / \mathrm{g}$ of gluconapin which resulted in $51.44 \mu \mathrm{mol} / \mathrm{g}$ of total Gls and $4.53 \mathrm{mg} / \mathrm{g}$ of erucic acid DM basis (Table 4).

Body weight and ADG at 6th wk of growing period were not affected by dietary RSM supplementation levels whereas at 13th wk of finishing and overall periods BW and ADG were decreased (linear, $\mathrm{p}<0.05$ ) by increasing dietary RSM supplementation levels in the growing-finishing diets (Table 5). Additionally, G/F ratio tended to decrease with increased dietary RSM supplementation levels during overall period (linear, $\mathrm{p}=0.07$ and quadratic; 0.08; Table 5). However, the pigs fed diets containing RSM levels showed no difference in $\mathrm{BW}$ and $\mathrm{ADG}$ of growing-finishing pigs compared to RSM 0 treatment.

Glucosinolates content in experimental diets was equivalent to $0,1.02,2.04,3.07$, or $4.09 \mu \mathrm{mol} / \mathrm{g}$ and erucic acid content was equivalent to $0,0.18,0.37,0.56$, or 0.70 $\mathrm{mg} / \mathrm{g}$ for $0 \%, 3 \%, 6 \%, 9 \%$, or $12 \%$ of $\mathrm{RSM}$ supplementation groups, respectively (Table 6). Average daily feed intake was not affected by dietary RSM supplementation levels (Table 6).

As dietary RSM level of finishing diet was increased, serum $\mathrm{T}_{3}$ and $\mathrm{T}_{4}$ concentrations at 13 th wk of finishing pigs were not affected among dietary RSM treatments (Table 7) whereas thyroid gland (linear, $\mathrm{p}<0.05$; Figure 1 ) and liver (linear, $\mathrm{p}<0.01$; Table 2) weight were increased by increasing dietary RSM supplementation levels at 13th wk of finishing pigs. Additionally, when pigs were fed diets containing $12 \%$ RSM showed higher liver weight at 13 th wk of finishing pigs compared to control diet $(p<0.01$; Figure 2).

Serum HDL cholesterol concentration at 13th wk of finishing period was increased as the dietary RSM supplementation levels increased (linear, $\mathrm{p}<0.05$; Table 8 )

Table 5. Influence of dietary rapeseed meal (RSM) on growth performance of growing and finishing pigs ${ }^{1}$

\begin{tabular}{|c|c|c|c|c|c|c|c|c|}
\hline \multirow{2}{*}{ Item } & \multicolumn{5}{|c|}{ Rapeseed meal level $(\%)^{2,3}$} & \multirow{2}{*}{ SEM } & \multicolumn{2}{|c|}{ p-value } \\
\hline & RSM 0 & RSM 3 & RSM 6 & RSM 9 & RSM 12 & & Lin. & Quad. \\
\hline \multicolumn{9}{|l|}{ Body weight (kg) } \\
\hline Initial & 29.86 & 29.88 & 30.02 & 29.95 & 30.03 & 1.323 & 0.134 & 0.745 \\
\hline 6th wk & 60.34 & 63.63 & 60.33 & 58.18 & 60.16 & 2.180 & 0.247 & 0.803 \\
\hline 13th wk & 105.48 & 109.34 & 104.86 & 103.18 & 100.63 & 2.360 & 0.049 & 0.276 \\
\hline \multicolumn{9}{|l|}{ Average daily gain (g) } \\
\hline 0 to 6 th wk & 725 & 803 & 721 & 671 & 717 & 23.987 & 0.209 & 0.812 \\
\hline 7 to 13 th wk & 920 & 933 & 909 & 924 & 825 & 14.257 & 0.044 & 0.110 \\
\hline Overall ( 0 to 13 th wk) & 830 & 873 & 822 & 803 & 775 & 14.530 & 0.040 & 0.277 \\
\hline \multicolumn{9}{|l|}{$\mathrm{G} / \mathrm{F}$ ratio } \\
\hline 0 to 6 th wk & 0.367 & 0.401 & 0.379 & 0.364 & 0.371 & 0.0056 & 0.403 & 0.234 \\
\hline 7 to 13 th wk & 0.305 & 0.310 & 0.303 & 0.310 & 0.287 & 0.0047 & 0.144 & 0.143 \\
\hline Overall ( 0 to 13 th wk) & 0.327 & 0.343 & 0.330 & 0.326 & 0.318 & 0.0034 & 0.066 & 0.082 \\
\hline
\end{tabular}

SEM, standard error of mean; Lin., linear; Quad., quadratic.

${ }^{1}$ A total of 120 crossbred pigs with an average initial body weight $29.94 \pm 0.06 \mathrm{~kg}$.

${ }^{2}$ RSM 0, basal diet; RSM 3, basal diet+3\% RSM; RSM 6, basal diet+6\% RSM; RSM 9, basal diet $+9 \%$ RSM; RSM 12, basal diet+12\% RSM.

${ }^{3}$ Gls content in the diets was equivalent to $0,1.02,2.04,3.07$, and $4.09 \mu \mathrm{mol} /$ for $0 \%, 3 \%, 6 \%$, $9 \%$, and $12 \%$ of RSM supplementation groups, respectively. 
Table 6. Influence of dietary rapeseed meal (RSM) on ingested glucosinolates (Gls) and erucic acid of growing-finishing pigs

\begin{tabular}{|c|c|c|c|c|c|c|c|c|}
\hline \multirow{2}{*}{ Item } & \multicolumn{5}{|c|}{ Rapeseed meal level (\%) } & \multirow{2}{*}{ SEM } & \multicolumn{2}{|c|}{ p-value } \\
\hline & RSM 0 & RSM 3 & RSM 6 & RSM 9 & RSM 12 & & Lin. & Quad. \\
\hline \multicolumn{9}{|l|}{ Growing period (0 to 6 th wk) } \\
\hline Feed intake (kg/d) & 1.98 & 2.01 & 1.91 & 1.86 & 1.94 & 63.332 & 0.333 & 0.546 \\
\hline Glsin feed $(\mu \mathrm{mol} / \mathrm{g})^{3}$ & - & 1.02 & 2.04 & 3.07 & 4.09 & & & \\
\hline Daily Gls intake $(\mu \mathrm{mol} / \mathrm{g})^{3}$ & - & 61.48 & 233.29 & 514.19 & 953.13 & & & \\
\hline Erucic acid in feed $(\mathrm{mg} / \mathrm{g})^{3}$ & - & 0.18 & 0.37 & 0.56 & 0.75 & & & \\
\hline Daily erucic acid intake $(\mathrm{mg} / \mathrm{g})^{3}$ & - & 361.62 & 705.22 & $1,042.16$ & $1,456.50$ & & & \\
\hline \multicolumn{9}{|l|}{ Finishing period ( 7 to 13 th wk) } \\
\hline Feed intake (kg/d) & 3.04 & 3.02 & 3.02 & 3.00 & 2.89 & 46.804 & 0.293 & 0.588 \\
\hline Gls in feed $(\mu \mathrm{mol} / \mathrm{g})^{3}$ & - & 1.02 & 2.04 & 3.07 & 4.09 & & & \\
\hline Daily Gls intake $(\mu \mathrm{mol} / \mathrm{g})^{3}$ & - & 92.35 & 369.53 & 828.07 & 1419.39 & & & \\
\hline Erucic acid in feed $(\mathrm{mg} / \mathrm{g})^{3}$ & - & 0.18 & 0.37 & 0.56 & 0.75 & & & \\
\hline Daily erucic acid intake $(\mathrm{mg} / \mathrm{g})^{3}$ & - & 543.24 & $1,117.03$ & $1,678.32$ & $2,169.00$ & & & \\
\hline
\end{tabular}

SEM, standard error of mean; Lin., linear; Quad., quadratic.

${ }^{1}$ RSM 0, basal diet; RSM 3, basal diet+3\% RSM; RSM 6, basal diet+6\% RSM; RSM 9, basal diet $+9 \%$ RSM; RSM 12, basal diet $+12 \%$ RSM.

${ }^{2}$ Gls content in the diets was equivalent to $0,1.02,2.04,3.07$, and $4.09 \mu \mathrm{mol} /$ for $0 \%, 3 \%, 6 \%, 9 \%$, and $12 \%$ of RSM supplementation groups, respectively.

${ }^{3}$ Calculated values.

Table 7. Influence of dietary rapeseed meal (RSM) on concentrations of serum triiodithyronine $\left(\mathrm{T}_{3}\right)$ and thyroxine $\left(\mathrm{T}_{4}\right)$ in finishing pigs

\begin{tabular}{|c|c|c|c|c|c|c|c|c|}
\hline \multirow{2}{*}{ Item } & \multicolumn{5}{|c|}{ Rapeseed meal level (\%) ${ }^{1,2}$} & \multirow{2}{*}{ SEM } & \multicolumn{2}{|c|}{ p-value } \\
\hline & RSM 0 & RSM 3 & RSM 6 & RSM 9 & RSM 12 & & Lin. & Quad. \\
\hline No. of finishing pigs & 6 & 6 & 6 & 6 & 6 & & & \\
\hline \multicolumn{9}{|c|}{ Triiodithyronine (ng/mL) } \\
\hline 13th wk & 0.98 & 1.02 & 0.93 & 1.25 & 1.08 & 0.056 & 0.289 & 1.000 \\
\hline \multicolumn{9}{|l|}{ Thyroxine (ng/mL) } \\
\hline 13 th wk & 6.30 & 6.20 & 6.65 & 7.27 & 6.47 & 0.211 & 0.361 & 0.494 \\
\hline
\end{tabular}

SEM, standard error of mean; Lin., linear; Quad., quadratic.

${ }^{1}$ RSM 0, basal diet; RSM 3, basal diet+3\% RSM; RSM 6, basal diet+6\% RSM; RSM 9, basal diet+9\% RSM; RSM 12, basal diet+12\% RSM.

${ }^{2}$ Gls content in the diets was equivalent to $0,1.02,2.04,3.07$, and $4.09 \mu \mathrm{mol} /$ for $0 \%, 3 \%, 6 \%, 9 \%$, and $12 \%$ of RSM supplementation groups, respectively.

whereas concentrations of serum total cholesterol and LDL cholesterol were not influenced by the dietary RSM supplementation in growing-finishing diets. Serum glucose concentration was not affected by the dietary RSM supplementation (Table 8).

In nutrient digestibility, the apparent total tract digestibility of dry matter tended to decrease when dietary
RSM supplementation level was increased (linear $=0.09$ ) whereas crude protein and crude fat were not affected by dietary RSM treatments. Fecal nitrogen tended to increase when $6 \%$ or $9 \%$ RSM was supplemented (quadratic, $\mathrm{p}=$ 0.06) whereas nitrogen retention and nitrogen digestibility were not influenced by dietary RSM supplementation (Table 9). Therefore, dietary RSM supplementation up to

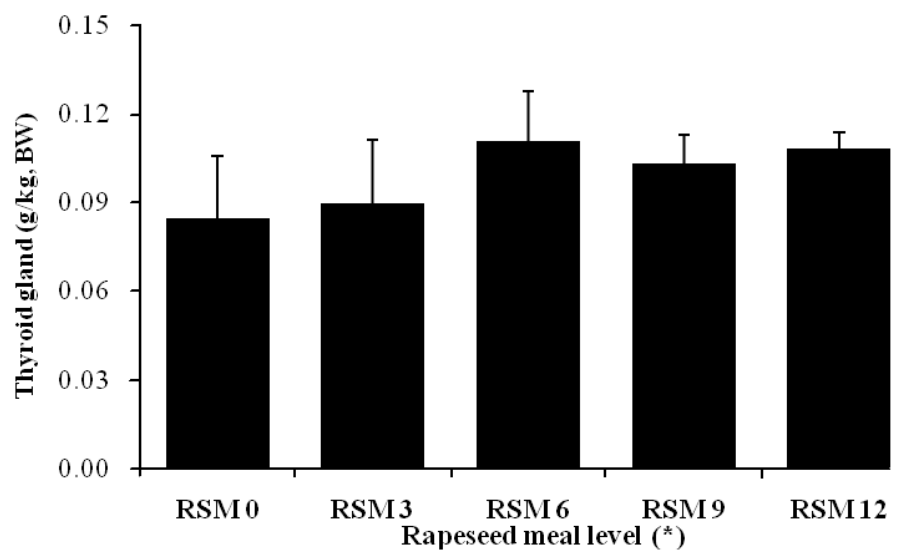

Figure 1. Influence of dietary rapeseed meal (RSM) utilization on thyroid gland weight at 13 th wk of finishing pigs $(* \operatorname{linear}, \mathrm{p}<0.05)$. 
Table 8. Influence of dietary rapeseed meal (RSM) on blood profiles of growing-finishing pigs

\begin{tabular}{|c|c|c|c|c|c|c|c|c|}
\hline \multirow{2}{*}{ Item } & \multicolumn{5}{|c|}{ Rapeseed meal level $(\%)^{1,2}$} & \multirow{2}{*}{ SEM } & \multicolumn{2}{|c|}{ p-value } \\
\hline & RSM 0 & RSM 3 & RSM 6 & RSM 9 & RSM 12 & & Lin. & Quad. \\
\hline No. of finishing pigs & 6 & 6 & 6 & 6 & 6 & & & \\
\hline \multicolumn{9}{|c|}{ Total cholesterol (mg/dL) } \\
\hline 13 th wk & 94.67 & 89.67 & 91.67 & 91.17 & 93.17 & 1.917 & 0.924 & 0.538 \\
\hline \multicolumn{9}{|c|}{ LDL cholesterol (mg/dL) } \\
\hline 13th wk & 65.17 & 57.67 & 54.50 & 54.00 & 55.83 & 1.820 & 0.111 & 0.193 \\
\hline \multicolumn{9}{|c|}{ HDL cholesterol (mg/dL) } \\
\hline 13th wk & 40.50 & 40.17 & 46.33 & 44.33 & 46.00 & 0.967 & 0.027 & 0.584 \\
\hline \multicolumn{9}{|l|}{ Glucose (mg/dL) } \\
\hline Initial & 63.00 & & & & & & & \\
\hline 6th wk & 87.33 & 83.33 & 93.83 & 81.00 & 82.50 & 1.745 & 0.351 & 0.416 \\
\hline 13th wk & 81.50 & 90.33 & 81.33 & 79.33 & 84.50 & 1.519 & 0.607 & 0.977 \\
\hline \multicolumn{9}{|c|}{ Blood urea nitrogen $(\mathrm{g} / \mathrm{dL})$} \\
\hline Initial & 12.40 & & & & & & & \\
\hline 6th wk & 9.12 & 8.23 & 6.97 & 7.92 & 7.48 & 0.308 & 0.083 & 0.195 \\
\hline 13th wk & 10.38 & 10.60 & 11.40 & 8.38 & 7.93 & 0.511 & 0.069 & 0.253 \\
\hline
\end{tabular}

SEM, standard error of mean; Lin., linear; Quad., quadratic; LDL, low density lipoprotein; HDL, high density lipoprotein.

${ }^{1}$ RSM 0, basal diet; RSM 3, basal diet+3\% RSM; RSM 6, basal diet+6\% RSM; RSM 9, basal diet $+9 \%$ RSM; RSM 12, basal diet $+12 \%$ RSM.

${ }^{2} \mathrm{Gls}$ content in the diets was equivalent to $0,1.02,2.04,3.07$, and $4.09 \mu \mathrm{mol} /$ for $0 \%, 3 \%, 6 \%, 9 \%$, and $12 \%$ of RSM supplementation groups, respectively.

$9 \%$ had no detrimental effects on nutrient digestibility and nitrogen retention in growing-finishing pigs.

Economic benefit was calculated by feed cost per weight gain (Table 10). The feed cost was numerically decreased when dietary RSM was supplemented during overall experimental period.

\section{DICUSSION}

A high level of Gls in the in the pig diet containing RSM resulted in poor growth performance when pigs were fed 1.3 to $2.8 \mu \mathrm{mol} / \mathrm{g}$ Gls (Bowland, 1975; Bell et al., 1991;
Tripathi and Mishra, 2007). In the results of the current study, BW, ADG, and G/F ratio were affected by dietary RSM supplementation levels up to $12 \%(4.09 \mu \mathrm{mol} / \mathrm{g}$ Gls $)$ in growing-finishing diets. Therefore, the result of the current study indicated that RSM supplementation in growing-finishing diets did not reduce growth performance when pigs were fed $9 \%$ of RSM or $3.07 \mu \mathrm{mol} / \mathrm{g}$ Gls was supplemented diet during growing-finishing periods.

When pigs were fed a RSM containing diet, thyroid gland and liver weight were increased at 13th wk finishing pigs. Additionally, pigs fed diets containing $12 \%$ of RSM showed higher liver weight at 13 th wk of finishing pigs

Table 9. Influence of dietary rapeseed meal (RSM) on nutrient digestibility in growing pigs ${ }^{1}$

\begin{tabular}{|c|c|c|c|c|c|c|c|c|}
\hline \multirow{2}{*}{ Item } & \multicolumn{5}{|c|}{ Rapeseed meal level $(\%)^{2,3}$} & \multirow{2}{*}{ SEM } & \multicolumn{2}{|c|}{ p-value } \\
\hline & RSM 0 & RSM 3 & RSM 6 & RSM 9 & RSM 12 & & Lin. & Quad. \\
\hline \multicolumn{9}{|c|}{ Nutrient digestibility (\%) } \\
\hline Dry matter & 93.98 & 93.53 & 91.52 & 87.95 & 92.04 & 0.813 & 0.087 & 0.246 \\
\hline Crude protein & 92.14 & 89.83 & 88.01 & 82.88 & 89.34 & 1.234 & 0.115 & 0.139 \\
\hline Crude fat & 73.28 & 74.22 & 69.21 & 68.87 & 77.41 & 2.539 & 0.880 & 0.404 \\
\hline \multicolumn{9}{|l|}{ Nitrogen retention $(\mathrm{g} / \mathrm{d})$} \\
\hline $\mathrm{N}$ intake & 13.90 & 12.31 & 14.04 & 13.79 & 13.83 & 0.170 & - & - \\
\hline Fecal N & 1.09 & 1.37 & 2.10 & 2.35 & 1.54 & 0.193 & 0.110 & 0.064 \\
\hline Urinary $\mathrm{N}$ & 7.70 & 7.74 & 7.83 & 8.23 & 8.11 & 0.361 & 0.652 & 0.996 \\
\hline $\mathrm{N}$ retention & 5.11 & 3.19 & 4.10 & 3.19 & 4.18 & 0.410 & 0.564 & 0.306 \\
\hline $\mathrm{N}$ digestibility $(\%)^{4}$ & 36.75 & 25.97 & 29.21 & 23.17 & 30.25 & 2.895 & 0.499 & 0.346 \\
\hline
\end{tabular}

SEM, standard error of mean; Lin., linear; Quad., quadratic.

${ }^{1} \mathrm{~A}$ total of 24 crossbred pigs were used from an average initial body weight of $31.14 \pm 0.36 \mathrm{~kg}$.

${ }^{2}$ RSM 0, basal diet; RSM 3, basal diet $+3 \%$ RSM; RSM 6, basal diet $+6 \%$ RSM; RSM 9, basal diet $+9 \%$ RSM; RSM 12, basal diet $+12 \%$ RSM.

${ }^{3} \mathrm{Gls}$ content in the diets was equivalent to $0,1.02,2.04,3.07$, and $4.09 \mu \mathrm{mol} /$ for $0 \%, 3 \%, 6 \%, 9 \%$, and $12 \%$ of RSM supplementation groups, respectively.

${ }^{4} \mathrm{~N}$ retention $=\mathrm{N}$ intake $(\mathrm{g})-$ Fecal $\mathrm{N}(\mathrm{g})-$ Urinary $\mathrm{N}(\mathrm{g})$. 
Table 10. Influence of dietary rapeseed meal (RSM) on economic benefits in growing-finishing diets

\begin{tabular}{|c|c|c|c|c|c|}
\hline \multirow{2}{*}{ Item } & \multicolumn{5}{|c|}{ Rapeseed meal level $(\%)^{1,2}$} \\
\hline & RSM 0 & RSM 3 & RSM 6 & RSM 9 & RSM 12 \\
\hline \multicolumn{6}{|l|}{ Feed cost (won/kg) } \\
\hline 0 to 6 th wk & 384 & 380 & 375 & 372 & 367 \\
\hline 7 th to 13 th wk & 361 & 356 & 352 & 348 & 344 \\
\hline Overall ( 0 to 13 th wk) & 373 & 368 & 364 & 360 & 355 \\
\hline \multicolumn{6}{|c|}{ Feed cost per weight gain (won/kg) } \\
\hline 0 to 6 th wk & 1,020 & 921 & 962 & 999 & 961 \\
\hline 7 th to 13 th wk & 1,228 & 1,195 & 1,210 & 1,167 & 1,252 \\
\hline Overall ( 0 to 13 th wk) & 1,140 & 1,075 & 1,106 & 1,107 & 1,124 \\
\hline Index $(\%)$ & 100.0 & 94.3 & 97.0 & 97.1 & 98.6 \\
\hline
\end{tabular}

${ }^{1}$ RSM 0, basal diet; RSM 3, basal diet+3\% RSM; RSM 6, basal diet+6\% RSM; RSM 9, basal diet+9\% RSM; RSM 12, basal diet+12\%RSM.

${ }^{2}$ Gls content in the diets was equivalent to $0,1.02,2.04,3.07$, and $4.09 \mu \mathrm{mol} /$ for $0 \%, 3 \%, 6 \%$, $9 \%$, and $12 \%$ of RSM supplementation groups, respectively.

compared to control diet. Increased thyroid gland and liver weight were associated with Gls breakdown product of nitriles (Mawson et al., 1994a). McKinnon and Bowland (1979) demonstrated that increased liver weight was observed when 2.0 to $4.0 \mu \mathrm{mol} / \mathrm{g}$ Gls were supplemented in pig diets.

The hydrolysis products of Gls are strongly related to goitrogenic substance subsequently interfere with iodine uptake and inhibit the synthesis of thyroid hormones $\mathrm{T}_{3}$ and $\mathrm{T}_{4}$ (Christison and Laarveld, 1981; Mawsonet al., 1994; Schöne et al., 1997). Mawson et al. (1994) reported that thyroid function observed in pigs fed high Gls RSM induced iodine deficiency which increased the serum $\mathrm{T}_{3}$ and $\mathrm{T}_{4}$ levels and liver weight because of Gls related iodine antagonistic effects. However, in this present experiment, there were no significant differences between control and dietary RSM supplementation treatments in the serum concentrations of $\mathrm{T}_{3}$ and $\mathrm{T}_{4}$. Serum total cholesterol and LDL cholesterol concentrations were not changed by RSM supplementation but serum HDL cholesterol concentration was increased. High intake of monounsaturated fatty acid resulted in increasing HDL cholesterol levels without changing LDL cholesterol levels (Jenkins et al., 2010). Additionally, it is known that RSM contained higher amount of monounsaturated $(52.0 \%$ vs $23.0 \%$ of the total fatty acid) than saturated (12.9\% vs $14.2 \%$ of the total fatty acid) or polyunsaturated $(33.0 \%$ vs $58.0 \%$ of the total fatty acid) fatty acids compared with SBM (Kracht et al., 2004; NRC, 2012).

Rapeseed meal has anti-nutritional factors such as tannins and phytic acid which reduces protein and amino acid digestion. Generally RSM has lower amino acid digestibility (87.0\% vs 90.4\%) than that of SBM (Bell, 1984; Green and Kiener, 1989). The fiber of rapeseed hulls decreased ileal digestibility of pigs and the high level of cell wall components in RSM reduces of protein availability. Also the decreased in $\mathrm{N}$ retention may reflect the reduced $\mathrm{N}$ digestibility coupled with reduced $\mathrm{N}$ intake with increasing dietary RSM levels (Patric et al., 2010).

Sauer et al. (1982) also indicated that the true ileal availabilities of amino acid were lower in RSM than SBM. When $9 \%$ of RSM was provided to growing-finishing pigs,

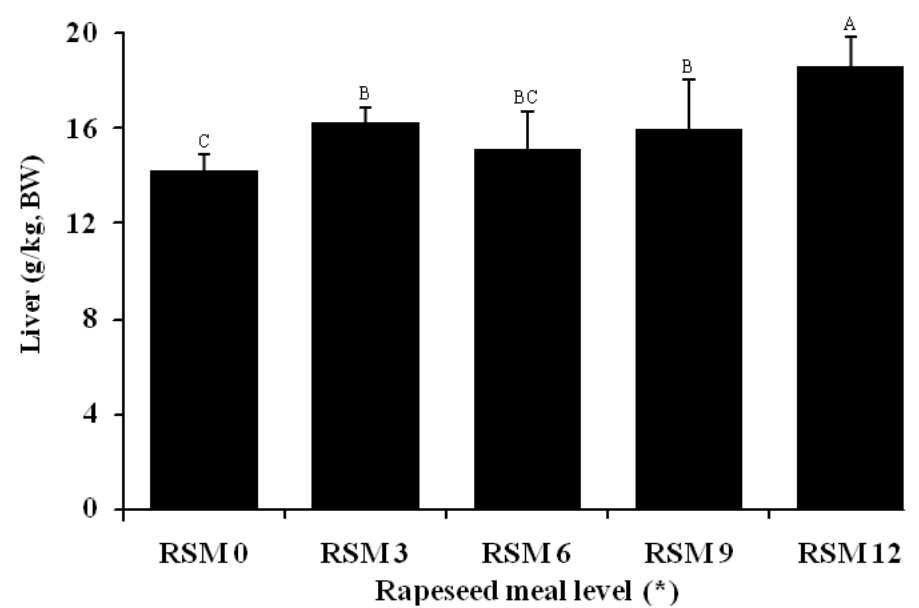

Figure 2. Influence of dietary rapeseed meal (RSM) utilization on liver weight at 13 th wk of finishing pigs $\left({ }^{*}\right.$ linear, $\left.p<0.01\right)$. ${ }^{A, B, C}$ means with different superscripts within the same row significantly differ $(\mathrm{p}<0.01)$. 
serum BUN concentration tended to decrease. In general, dietary lysine level was related to BUN concentration and nitrogen retention (Eggum, 1970) but nitrogen retention and nitrogen digestibility were not affected by dietary RSM supplementation.

Economic benefit was increased when 9\% of RSM was supplemented in growing-finishing diets because RSM is a cost saving ingredient in pig diets (Quiniou et al., 2012).

\section{IMPLICATIONS}

Several impacts of increasing RSM levels in growingfinishing diets were observed in growth performance and organ weight; decreased $\mathrm{BW}, \mathrm{ADG}$, and $\mathrm{G} / \mathrm{F}$ ratio when RSM was provided up to $12 \%$. Increased thyroid gland as well as liver weight were observed as dietary RSM supplementation levels increased and serum HDL cholesterol concentration was increased by dietary RSM supplementation. However, RSM could be supplemented up to $9 \%(3.07 \mu \mathrm{mol} / \mathrm{g} \mathrm{Gls})$ in growing-finishing pig diets without any detrimental effect on growth performance.

\section{ACKNOWLEDGMENTS}

This research was supported by Nonghyup feed Inc. (Project No. 0569-20120010), Nonghyup feed Inc., Republic of Korea.

\section{REFERENCES}

AOAC. 1990. Official Methods of Analysis. 15th ed. Association of Official Analytical Chemists, Arlington, VA, USA.

Bell, J. M. 1984. Nutrients and toxicants in rapeseed meal: A review. J. Anim. Sci. 58:996-1010.

Bell, J. M., M. O. Keith, and D. S. Hutcheson. 1991. Nutritonal evaluation of very low glucosinolate canola meal. Can. J. Anim. Sci. 71:497-506.

Bowland, J. P. 1975. Evaluation of low glucosinolate-low erucic acid rapeseed meal as the protein supplement foryoung growing pigs, including effects on blood serum constituents. Can. J. Anim. Sci. 55:409-416.

Christison, G. I. and B. Laarveld. 1981. Thyroid hormone response to thyrotropin releasing hormone by pigs fed canola, rapeseed or soybean meals. Can. J. Anim. Sci. 61:1023-1029.

Eggum, B. O. 1970. Blood urea measurement as a technique for assessing protein quality. Br. J. Nutr. 24:983-988.

Food Standards Australia New Zealand. 2003. Erucic acid in food: A toxicological review and risk assessment. Tech. Report Series 21:1448-3017.

Green, S. and T. Kiener. 1989. Digestibilities of nitrogen and amino acids in soya-bean, sunflower, meat and rapeseed meals measured with pigs and poultry. Anim. Prod. 48:157-179.
International Standards Organisation (ISO). 1992. Rapeseed: Determination of glucosinolates content. ISO 9167-1. Geneva, Switzerland.

Kracht, W., S. Dänicke, H. Kluge, K. Keller, W. Matzke, U. Hennig, and U. Schumann. 2004. Effect of dehulling of rapeseed on feed value and nutrient digestibility of rape products in pigs. Arch. Anim. Nutr. 58:389-404.

Mawson, R., R. K. Heaney, M. Piskula, and H. Kozlowska. 1993a. Rapeseed meal-glucosinolates and their antinutritional effects 1. Rapeseed production and chemistry of glucosinolates. Nahrung. 37:131-140.

Mawson, R., R. K. Heaney, Z. Zdunczyk, and H. Kozlowska. 1993b. Rapeseed meal-glucosinolates and their antinutritional effects: 2. Flavour and paltability. Nahrung 37:336-344.

Mawson, R., R. K. Heaney, Z. Zdunczyk, and H. Kozlowska. 1994. Rapeseed meal-glucosinolates and their antinutritional effects: 4. Goitrogenicity and internal organs abnormalities in animals. Nahrung 38:178-191.

McKinnon, P. J. and J. P. Bowland. 1979. Effects of feeding low and high glucosinolate rapeseed meals and soybean meal on thyroid function of young pigs. Can. J. Anim. Sci. 59:589-596.

National Research Council. 1998. Nutrient Requirements of Swine, 10th ed. National Academy Press, Washington, DC, USA.

National Research Council. 2012. Nutrient Requirements of Swine, 11th ed. National Academy Press, Washington, DC, USA.

Noblet, J. and G. L. Goff. 2001. Effect of dietary fibre on the energy value of feeds for pigs. Anim. Feed Sci. Technol. 90: $35-52$.

Patrick, M., C. O'Shea, S. Figat, and J. V. O'Doherty. 2010. Influence of incrementally substituting dietary soya bean meal for rapeseed meal on nutrient digestibility, nitrogen excretion, growth performance and ammonia emissions from growingfinishing pigs. Arch. Anim. Nutr. 64:412-424.

Quiniou, N., A. Quinsac, K. Crépon, J. Evrard, C. Peyronnet, A. Bourdillon, E. Royer, and M. Etienne. 2012. Effects of feeding $10 \%$ rapeseed meal (Brassica napus) during gestation and lactation over three reproductive cycles on the performance of hyperprolific sows and their litters. Can. J. Anim. Sci. 92:513524.

SAS Institute Inc. 2009. SAS/STAT User's Guide, Version 9.2. SAS Institute Inc., Cary, NC, USA.

Sauer, W. C., R. Cichon, and R. Misir. 1982. Amino acid availability and protein quality of canola and rapeseed meal for pigs and rates. J. Anim. Sci. 54:292-301.

Schöne, F., M. Leiterer, G. Jahreis, and B. Rudolph. 1997. Effect of rapeseed feedstuffs with different glucosinolate content and iodine administration on gestating and lactating sow. J. Vet. Med. A. 44:325-339.

Smit, M. N., R. W. Seneviratne, M. G. Young, G. Lanz, R. T. Zijlstra, and E. Beltranena. 2014. Feeding increasing inclusions of canola meal with distillers dried grains and solubles to growing-finishing barrows and gilts. Anim. Feed. Sci. Technol. 189:107-116.

Tripathi, M. K. and A. S. Mishra. 2007. Glucosinolates in animal nutrition: A review. Anim. Feed. Sci. Technol. 132:1-27. 